\title{
LET'S FOCUS: ATTENTION-DEFICIT/HYPERACTIVITY DISORDER (ADHD) IN THE ADULT
}

C. Leite Rodrigues1, L. Castanheira1, E. Fernandes1, C. Cordovil2, I. Duarte e Silva1.

1 Centro Hospitalar Lisboa Norte, Neurosciences Department - Psychiatry and Mental Health, Lisbon, Portugal.

2Centro Hospitalar Lisboa Norte, Pediatrics Department - Psychiatry of Childhood and Adolescence, Lisbon, Portugal.

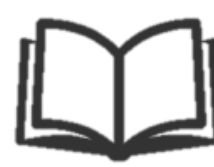

\section{INTRODUCTION}

ADHD was originally described in children but is now recognized to persist into adulthood for some patients, although is still underdiagnosed. Diagnostic criteria include adult-specific symptoms and behavioural manifestations, which may aid in the diagnosis. However, ADHD remains a complex and challenging condition due to symptom overlap with other psychiatric disorders that might be comorbid.

\section{AIMS}

To describe the characteristics of adult ADHD and address management strategies and pharmacological treatment controversies.

\section{METHODS}

Non-systematic review of the literature published between 2009 and 2019 using PubMed database, with the terms "ADHD", "attention deficit", "hyperactivity" and "adult". The articles found were selected by the authors according their scientific relevance for the present aim.

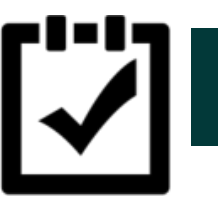
RESULTS

It was believed that attention deficit and hyperactivity disorder (ADHD) was outgrown by the end of adolescence and the beginning of adulthood. The diagnostic criteria in the DSM-V list ADHD under the category neurodevelopmental disorders, rather than the category of disorders first diagnosed in infancy, childhood or adolescence as it was in the DSM-IV. This changes will increase the diagnosis of adult ADHD.

Prevelance is estimated to be $5-7 \%$ in children, with male:female ratio of at least 3:1, 2/3 of children diagnosed with ADHD experience as adults ADHD symptoms that impair their functionality and adult ADHD prevalence rate ranges from $2.5 \%$ to $5.2 \%$. Inattentive subtype is the most prevalent in adults affecting nearly half of the patients. Symptom presentation vary between children and adults (fig. 1).
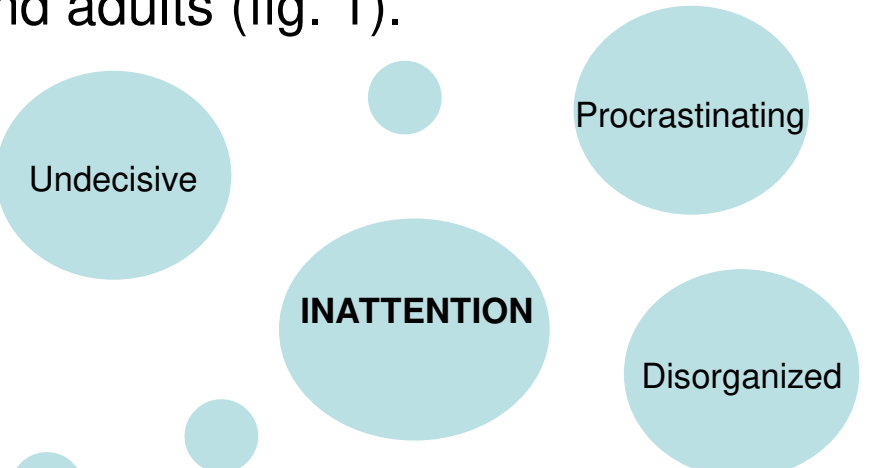

Adult ADHD

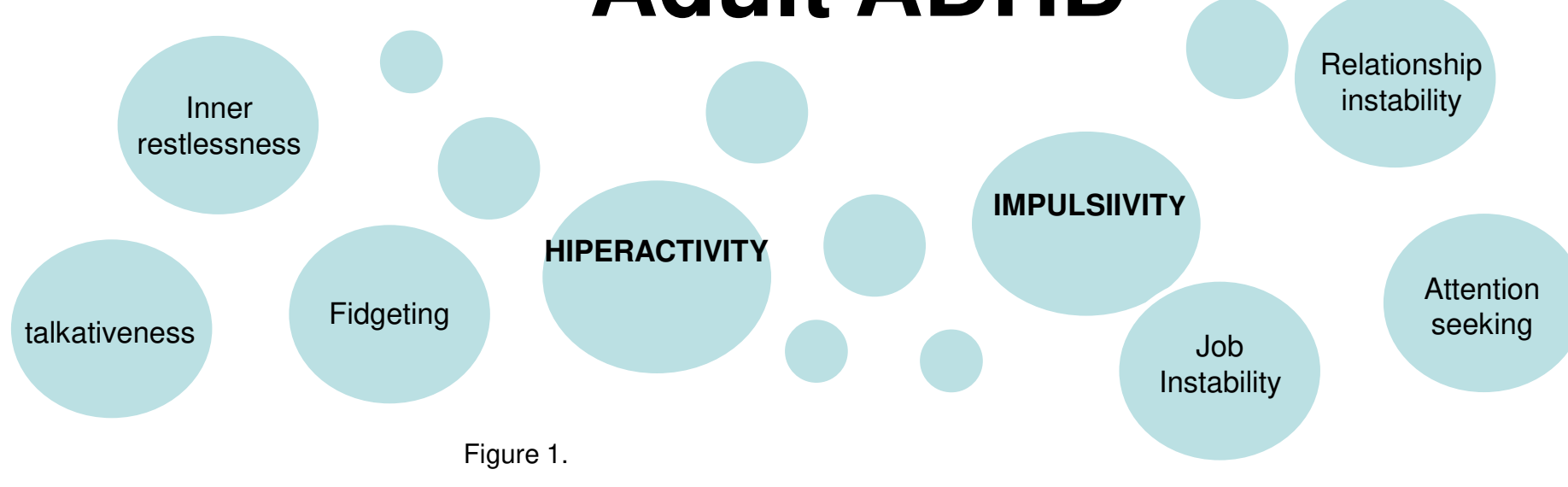

\section{DISCUSSION \& CONCLUSIONS}

- Adult ADHD patients often suffer from unemployment, financial difficulties and an unsuccessful personal life.

- Longitudinal family histories provide valuable information that diagnoses and differentiates this condition from other psychiatric comorbidities.

- There are major differences between childhood and adult ADHD, requiring individualized strategies for each patient. Adult ADHD should be primarily treated with pharmacological intervention assisted by cognitive-behavioural therapy.
- $\mathrm{ADHD}$ is a disorder expressed primarily by the distribution of attention over many stimuli without the ability to focus on a single stimulus for a period of time.

- Due to constant demands of adult life, a long-acting medication formulation is often preferable to a short acting one. Important concerns with medication side effects and dependence should be considered carefully before prescribing stimulants and adult-specific guidelines may improve ADHD treatment in this patient group.

- There is a need for further collaboration between clinicians to better understand and manage this condition

\section{REFERENCES:}

[1] Zalsman, G., \& Shilton, T. (2016). Adult ADHD: A new disease?. International Journal Of Psychiatry In Clinical Practice, 20(2), 70-76.

[2] Young, J., \& Goodman, D. (2016). Adult AttentionDeficit/Hyperactivity Disorder Diagnosis, Management, and Treatment in the <em>DSM-5</em> Era. The Primary Care Companion For CNS Disorders.
[3] Jain, R., Jain, S., \& Montano, C. (2017). Addressing Diagnosis and Treatment Gaps in Adults With AttentionDeficit/Hyperactivity Disorder. The Primary Care Companion For CNS Disorders, 19(05).

[4] Wu, J., Xiao, H., Sun, H., Zou, L., \& Zhu, L. (2012). Role of Dopamine Receptors in ADHD: A Systematic Metaanalysis. Molecular Neurobiology, 45(3), 605-620. 